\title{
Is Percutaneous Ethanol Injection Therapy Still Effective for Hepatocellular Carcinoma in the Era of Radiofrequency Ablation?
}

\author{
Jung Hyeok Kwon \\ Department of Radiology, Keimyung University School of Medicine, Daegu, Korea
}

Percutaneous ethanol injection (PEI) therapy has been replaced by more-effective thermal ablation techniques that have lower local recurrence rates. However, PEI therapy remains useful in certain settings. Since PEI can be performed in any portion of the liver, PEI therapy can be valuable when tumors are located in close proximity to intestinal loops or other positions that are risky for thermal local ablative techniques. PEI therapy is also valuable in other situations where radiofrequency ablation (RFA) is difficult, including technically difficult masses that are not detected with ultrasound (US), are located in the hepatic dome, in the subcapsular area, and exophytically, or are surrounded by large vessels. PEl therapy contributes to combination therapy with transcatheter arterial chemoembolization or RFA in advanced-stage hepatocellular carcinoma ( $\mathrm{HCC})$, and also to the treatment of large $\mathrm{HCC}$ or extrahepatic metastasis from HCC. These roles of PEI therapy should be stressed for the treatment of HCCs in appropriate clinical situations. This comprehensive review of articles related to PEI therapy illustrates the recent role and indications of this therapy, which is currently valuable for HCC in the era of RFA. (Gut Liver 2010;4(Suppl. 1):S105-112)

Key Words: Hepatocellular carcinoma; Local ablation therapy; Percutaneous ethanol injection therapy

\section{INTRODUCTION}

Because of the multifocal nature of hepatocellular carcinoma (HCC) and the relatively high complication rate associated with surgery, locoregional therapy has gained an increasing role in the treatment of HCC. ${ }^{1-5}$ Non-surgical therapy of HCC is based on radiofrequency ablation (RFA), percutaneous ethanol injection (PEI) therapy, and transcatheter arterial chemoembolization (TACE), with RFA and PEI therapy being effective in small HCC and TACE being the method of choice in advanced tumor stage. ${ }^{1-3}$ RFA enables more effective tumor necrosis in fewer treatment sessions, albeit with a higher rate of complications than does PEI therapy. ${ }^{1,6,7}$ Because of its high local recurrence rate, PEI therapy has been replaced by newer and more effective thermal ablation techniques. ${ }^{6}$ It is reasonable to reserve PEI therapy for HCCs that are difficult to approach with RFA or located in areas where RFA is considered unsafe. ${ }^{8}$ Because PEI carries no risk of damage to the remaining parenchyma, this therapy is safer than any other treatment modality of HCC., ${ }^{3,9}$ Also, because combinations of RFA, PEI therapy, and TACE may produce a synergistic effect, efficacy of local ablation in HCC can be improved by combining different interventional therapies. ${ }^{4,5,8}$ In addition, PEI therapy has it possible to treat medium and large HCCs under conscious sedation. ${ }^{10,11}$ Moreover, this therapy could be performed in limited cases of extrahepatic metastasis from HCC. ${ }^{12,13}$

This article aims to provide us with a comprehensive review of PEI therapy currently valuable for HCC in the era of RFA. The recent role of PEI therapy is highlighted.

\section{CHOICE AMONG VARIOUS LOCOREGIONAL THERAPIES}

The proper selection of locoregional therapy depends on careful evaluation of the tumor and liver function status as well as updated knowledge of the results of various

\footnotetext{
Correspondence to: Jung Hyeok Kwon

Department of Radiology, Keimyung University School of Medicine, 126 Dalseong Street, Dongsan-dong, Jung-gu, Daegu 700-712, Korea

Tel: +82-53-250-7770, Fax: +82-53-250-7766, E-mail: kjh2603@dsmc.or.kr DOI: $10.5009 /$ gnl.2010.4.S1.S105
} 
treatment modalities. ${ }^{8}$ For patients with single HCC of $<5 \mathrm{~cm}$ in diameter or 3 or less nodules of $<3 \mathrm{~cm}$ in diameter, local ablation should be offered when surgical resection or transplantation is not possible. For patients with large or multifocal HCCs (four or more) and reasonable liver function, TACE is considered an effective treatment. However, for patients with HCCs larger than 5 $\mathrm{cm}$, combination therapy would be better. For patients with poor hepatic function at risk with TACE, local ablation should be considered. ${ }^{8}$

Currently, RFA is a first-line treatment of the major local ablative techniques for the treatment of HCC, preferably smaller than $3 \mathrm{~cm}$, which is not suited to resection or transplantation. ${ }^{5,6}$ However, PEI therapy remains useful in select settings, particularly for the ablation of unresectable tumors whose locations make them at risk with other thermoablative modalities. Moreover, PEI therapy is also valuable in other situations difficult to ablate with RFA, including technically difficult masses, which are not detected with ultrasound (US), are located in the hepatic dome, in the subcapsular area, and exophytically, or are surrounded by large vessels. ${ }^{3,9}$ We recommend RFA in the majority of HCCs smaller than $3 \mathrm{~cm}$ but consider PEI therapy and TACE complementary and use them according to the features of the disease, that is, size, number, location, margins, presence of satellites or portal thrombosis, and the response. ${ }^{3,9}$ PEI therapy as well as segmental TACE will continue to have a valuable complementary role in patients with early-stage HCC. ${ }^{3,6,8}$ Appropriate use of each treatment technique can only be done when the therapeutic strategy is decided by a multidisciplinary team and is tailored to the individual patient and to the features of the disease. ${ }^{6,8}$

\section{UNDERSTANDING OF PEI THERAPY AND HIGH-DOSE PEI THERAPY}

There are three different approaches towards PEI therapy in HCC. ${ }^{1,3}$ Low-dose multisession technique (conventional technique) is generally used for single HCC of $<5 \mathrm{~cm}$ in diameter or 3 or less nodules of $<3 \mathrm{~cm}$ in diameter, and aims for patient acceptance and a low rate of side effects. It is performed on an outpatient basis with an injected volume per session of 2-10 $\mathrm{mL}^{8}$ Single-session procedure is usually performed under general anesthesia, and adopted for intermediate HCCs, single or multiple, that do not occupy more than $30 \%$ of the hepatic volume and with no neoplastic thrombosis in the main portal branches or in the hepatic veins. A markedly higher amount of ethanol is needed to achieve complete tumor necrosis in a single session. The injection volume can be approximated from the lesion diameter by calculating the lesion volume as follows: $V=4 / 3 \pi(r+0.5)^{3}$. 1,3-5,9 The other technique, high-dose technique (tolerable amount more than $10 \mathrm{~mL}$ per session without general anesthesia) is intermediate between multi-session and single session, and covers indications of multi-session and those of single session. The total of calculated amount of ethanol is divided by several sessions and injected as much as possible with tolerable amount per session. ${ }^{14}$

It is generally agreed that patients with HCCs $3 \mathrm{~cm}$ or smaller and three or fewer in number are the best candidates for PEI therapy, although many centers perform PEI therapy for HCCs up to $5 \mathrm{~cm}^{8}{ }^{8}$ Tumor size larger than 5 $\mathrm{cm}$ has been traditionally regarded to be unsuitable for PEI therapy. However, for HCCs of 5 to $10 \mathrm{~cm}$ single-session treatment under general anesthesia have been used in some institutions. But this technique requires general anesthesia and produces higher complication rates. ${ }^{8}$ If one chooses to inject large quantity of ethanol of tolerable amount more than $10 \mathrm{~mL}$ per session without general anesthesia, the patient will be tolerated and the number of sessions will be decreased with effective tumor necrosis. ${ }^{14}$ In my experience, patients could easily tolerate injections of $10-30 \mathrm{~mL}$ of ethanol per session with only Demerol injection if necessary. So a nodule $3 \mathrm{~cm}$ in diameter can be treated in two sessions at the most. Nodules up to $2 \mathrm{~cm}$ in diameter can be treated in one session. Standard percutaneous ablation therapy is performed with real-time US-guidance. However, problems remain with percutaneous ablation therapy carried out under USguidance. Because gases from destructed tissue even with small amount of ethanol obscure a needle tip position at US, to inject high-dose ethanol continuously under the US-monitoring may give an anxiety to a performer. And so safe high-dose injection would be possible under computed tomography (CT)-guidance. ${ }^{15}$

\section{TECHNIQUE OF CT-GUIDED HIGH-DOSE PEI THERAPY}

I will illustrate the technique of CT-guided high-dose PEI therapy (Fig. 1). The selected body position is mostly supine, or rarely prone or lateral decubitus, depending on the proximity of the lesion to the abdominal wall. After a needle marker grid is applied to the skin, noncontrast CT scan with a 5-mm section thickness is performed to determine the optimal skin entry point and the safest route to tumor masses for bypassing the dangerous critical organ. The angle and depth of needle puncture are determined using the geometric relationship between the target lesion and the skin puncture point on the CT 

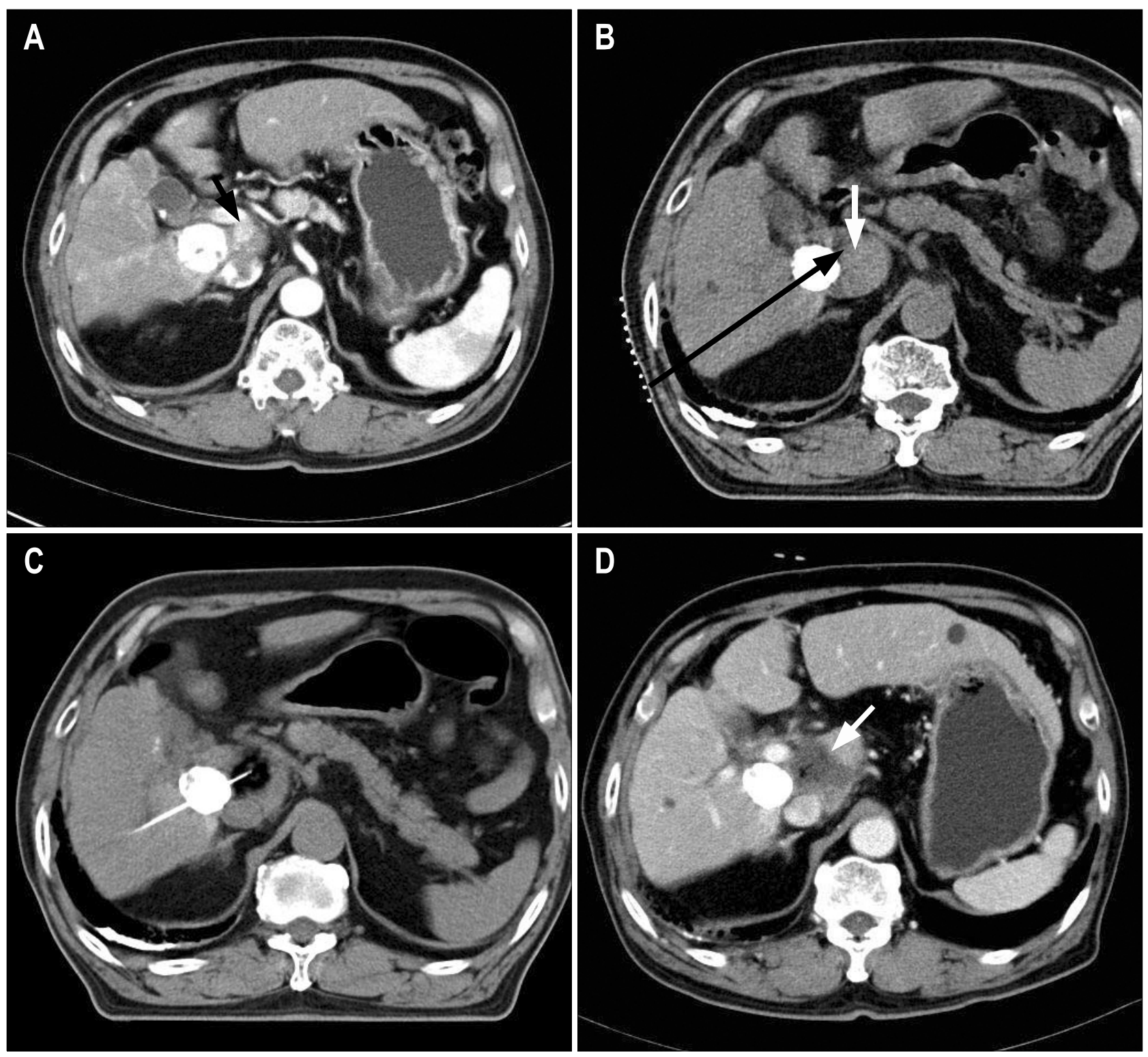

Fig. 1. Method of computed tomography (CT)-guided high-dose percutaneous ethanol injection (PEI) therapy in a 76-year-old man with viable hepatocellular carcinoma (HCC) in the caudate lobe of the liver in spite of repeated transcatheter arterial chemoembolization. Radiofrequency ablation was not suitable for this mass since it abutted the portal-vein trunk and was invisible in ultrasound. (A) Contrast-enhanced CT image shows an enhanced mass (arrow) in the caudate lobe of the liver in the vicinity of a mass with compact Lipiodol uptake. (B) The procedure. In a supine body position, place a needle marker grid for a possible entry point on the skin. Determine the angle and depth of the needle puncture (black arrow) using the geometric relationship between the target lesion (white arrow) and the skin puncture point on a CT image. (C) Confirm needle side holes within the nodule. Instill ethanol and monitor the needle position, the diffusion of ethanol within the nodule, and whether ethanol leaks into adjacent veins or structures, by performing noncontrast CT scans after every 3-mL injection. Administer ethanol until the region with markedly low attenuation becomes at least as large as the mass. Ignore small leaks of ethanol into surrounding normal tissues or structures. (D) Contrast-enhanced CT image obtained 1 day after PEI therapy shows complete necrosis of HCC (arrow).

image. We use 21-gauge or 22-gauge PEI therapy needles (Hakko, Tokyo, Japan) that are 15- or 20-cm long and have a closed conical tip and three or six side holes. The standard total dose of injected ethanol is calculated according to the following equation: $V=4 / 3 \pi(r+0.5)^{3}$, where $V$ is the volume of ethanol in milliliters and $r$ is the radius of a nodule in centimeters. The addition of 0.5 to the radius is designed to achieve the necrosis of a rim of nontumorous tissue around the tumor. Total dose should be divided into tolerable amount per session. The diffusion of ethanol in the nodule is monitored with CT-guidance by performing noncontrast CT scan after ev- 
ery 3-mL ethanol injection. Because injected ethanol shows markedly low attenuation at CT image, we administer ethanol until the size of the markedly low attenuation becomes larger than or equal to the size of the nodule. ${ }^{16}$ We inject ethanol as much as possible with tolerable amount per session. In our study, $10-30 \mathrm{~mL}$ of absolute ethanol was injected per session. If we find significant ethanol leaks into surrounding veins or surrounding structures, we stop injecting ethanol. Demerol $50 \mathrm{mg}$ is intravenously injected in patients who complain of severe pain during or after ablation therapy. To prevent reflux of ethanol along the needle tract and leakage into the peritoneal cavity, the needle is aspirated with negative pressure and left in place for 20-30 seconds after completion of the injection and then slowly withdrawn. PEI sessions are performed daily if possible, to decrease hospital stay. Entire portion of the tumor should be changed to black or hypoattenuating areas. ${ }^{12}$ The remaining, unchanged areas within the mass represent residual viable tumor. At next sessions, one should preferentially inject ethanol into unchanged areas under CT-guidance. ${ }^{4}$

\section{ROLE OF PEI THERAPY IN THE ERA OF RFA}

\section{HCCs at risk or difficult to treat with RFA}

As PEI therapy can be performed in any portion of the liver, PEI therapy remains useful for the ablation of unresectable tumors whose locations make them at risk or difficult to treat with other thermoablative modalities. Our experience of PEI therapy for 124 patients with 142 HCCs which were $3 \mathrm{~cm}$ or smaller and at risk or difficult to treat with RFA showed $84 \%, 74 \%, 68 \%$, and $56 \%$ at the 1-, 2-, 3-, and 5-year rates of survival, respectively (unpublished data; presented at oncology symposium, Radiologic Society of North America, 2005). As compared with other articles about PEI therapy for HCCs in favorable location, similar therapeutic results were obtained. ${ }^{1-9}$ Of note is the report of Kuang et al. ${ }^{10}$ that the effectiveness and complication rate of PEI therapy for treating tumors in unfavorable location for RFA were similar to those of PEI therapy for treating tumors in favorable location for RFA.

\section{1) HCC at risk with RFA}

Tumors close $(<1 \mathrm{~cm})$ to other vital adjacent structures such as main biliary ducts, gallbladder, stomach, bowel loop (especially when hepato-intestinal adhesions are present), kidney, and diaphragm require careful consideration as thermal damage to these organs has been described, exclusively after percutaneous RFA procedures. ${ }^{5,6}$ However, as PEI therapy can be performed in any portion of the liver, it is a valuable therapy in these situations.

\section{2) HCC not detected with US}

Sometimes some nodules cannot be detected with US, such as nodules that are located in the hepatic dome, in the deep region of the liver, and on the surface, or small nodules that are $1.0 \mathrm{~cm}$ or smaller (Figs. 1 and 2). US is not considered ideal in complex anatomic tumor locations. CT- and MR-guidance may be even more advantageous as both techniques offer a significantly more panoramic view than US and with modern fluoroscopy modes both techniques allow for a virtually ideal targeting of all areas of the liver. ${ }^{1,11}$ CT-guided PEI therapy is safe and effective for the treatment of HCC not detectable with US. ${ }^{15,17,18}$ CT-guided RFA may solve this situation.

\section{3) HCC located subcapsularly or exophytically}

Subcapsular tumors can be easily ablated without difficulties in contrast to RFA. When we puncture a mass after crossing normal parenchyma apart from the mass, the mass can be safely ablated. ${ }^{4}$ Exophytic tumor can be ablated after crossing normal parenchyma from opposite side. When a window of normal parenchyma is not seen, a direct puncture into an exophytic mass might be an only choice.

\section{4) HCC abutting on greater vessels}

RFA cannot ablate the periphery of the tumor and the cuff of normal hepatic tissue, when the tumor is surrounded by large vessels, because of the heat-sink effect of flowing blood. ${ }^{19}$ In contrast, PEI therapy can ablate safely HCCs without difficulty, in case of masses abutting on or surrounded by large vessels (Figs. 1 and 2). HCCs surrounded by large vessels can be easily ablated without a difference of therapeutic results, as compared with HCCs not surrounded by large vessels or vital structures.

\section{Combination therapy}

RFA, PEI therapy, or TACE should be considered complementary rather than competitive each other. Similarly, transarterial and local ablative therapies should not be regarded as mutually exclusive, because combinations of the two may produce a synergistic effect. ${ }^{4,5,8}$ The combination of TACE and PEI therapy, TACE and RFA, or RFA and PEI therapy is more effective than each of the monotherapies in preventing incomplete necrosis, especially in advanced disease or when tumors are in close proximity to large vessels. ${ }^{1}$ Using combination therapies with TACE, RFA, and PEI therapy, it is also suited for tumors up to $5-7 \mathrm{~cm}$, especially effective for tumors $3-5 \mathrm{~cm}$. 

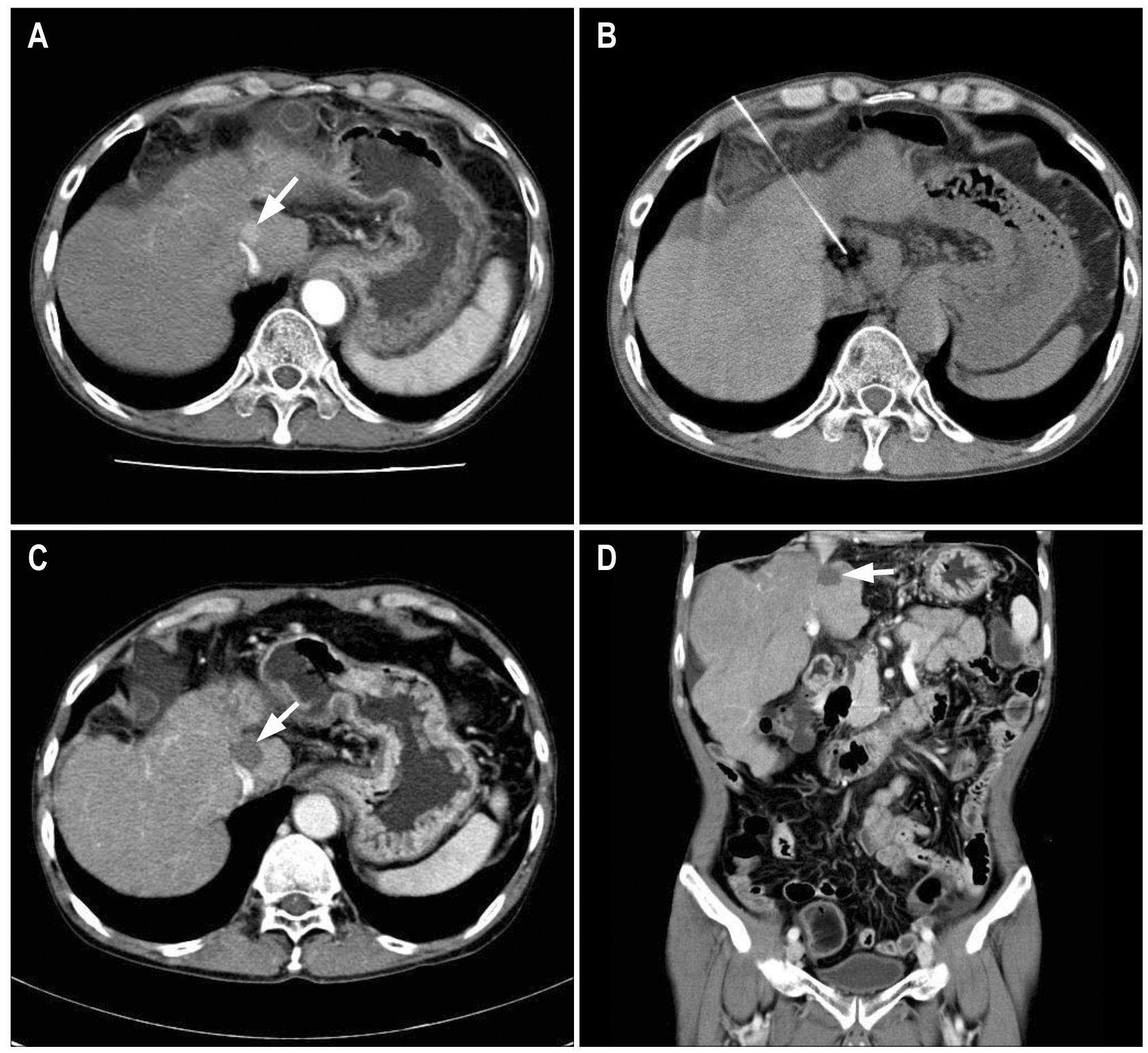

Fig. 2. Computed tomography (CT)-guided high-dose percutaneous ethanol injection (PEI) therapy in a 53-year-old man with a small hepatocellular carcinoma in the caudate of the liver that abutted on inferior vena cava and was invisible in ultrasound. (A) Contrast-enhanced CT image shows a mass in the caudate lobe of the liver (arrow). (B) CT-guided PEI therapy was performed for this mass at two sessions, with $20 \mathrm{~mL}$ of $99 \%$ ethanol being injected during each session. (C, D) Contrast-enhanced axial (C) and coronal (D) abdominal CT images obtained 3 years after PEI therapy show complete necrosis of this mass (arrows).

Thus, the use of multimodal treatment regimens needs to be expanded. ${ }^{1}$

\section{1) Combination therapy with TACE}

The combination of TACE and PEI therapy has been used to overcome the limitations of each for the treatment of large HCCs. The greatest drawback of PEI therapy in the treatment of HCC is represented by the difficulty to ablate tumors larger than $5 \mathrm{~cm}$. In large lesions, ethanol diffusion is often inhomogeneous and incomplete because it is impeded by the texture of the tumor and by intratumoral septa. In large HCCs that are pretreated with TACE, the tumor resistance to perfusion with ethanol is markedly decreased, and any intratumoral septa are usually disrupted as a result of the necrosis induced by TACE, which facilitates the diffusion of the ethanol and makes it possible to inject large volume of ethanol into the lesion. Also, the washout of ethanol is delayed with the TACE-derived fibrous wall around the lesion after arterial embolization, resulting in longer retention and tumoricidal effect. ${ }^{8,20,21}$ Kirikoshi et al. $^{21}$ reported that in terms of the effect on the survival period, combined 
TACE+PEI or RFA therapy was more effective than TACE, PEI, or RFA monotherapy. Compared with repeated TACE, PEI therapy after single TACE is more effective in large HCC and avoids problems such as the development of collateral blood supply to the tumor, resistance to cytotoxic drugs, and progressive liver damage. ${ }^{9,20}$

\section{2) Combination therapy with RFA}

The combination of RFA and PEI therapy results in a larger volume of coagulation necrosis than does RFA alone, probably because of the effect of hot ethanol in extending tissue necrosis, diffusion of ethanol into areas not reached by radiofrequency energy, and reduction of the heat sink effect. The combination of RFA and PEI therapy in the management of HCC in high-risk locations has a higher primary effectiveness rate than does RFA alone.,11 Zhang et al. ${ }^{22}$ reported that the survival curve for the RFA-PEI group was better than that for the RFA-only group with 3.1-5.0-cm tumors, but not for those with 3.0 $\mathrm{cm}$ or smaller or $5.1-7.0 \mathrm{~cm}$ tumors.

\section{Ablation or volume debulking of large HCC}

It has been possible to treat large HCCs with large quantity of ethanol. This procedure is valuable in combination with TACE for this purpose. PEI therapy with high-dose ethanol may be a valid alternative in patients
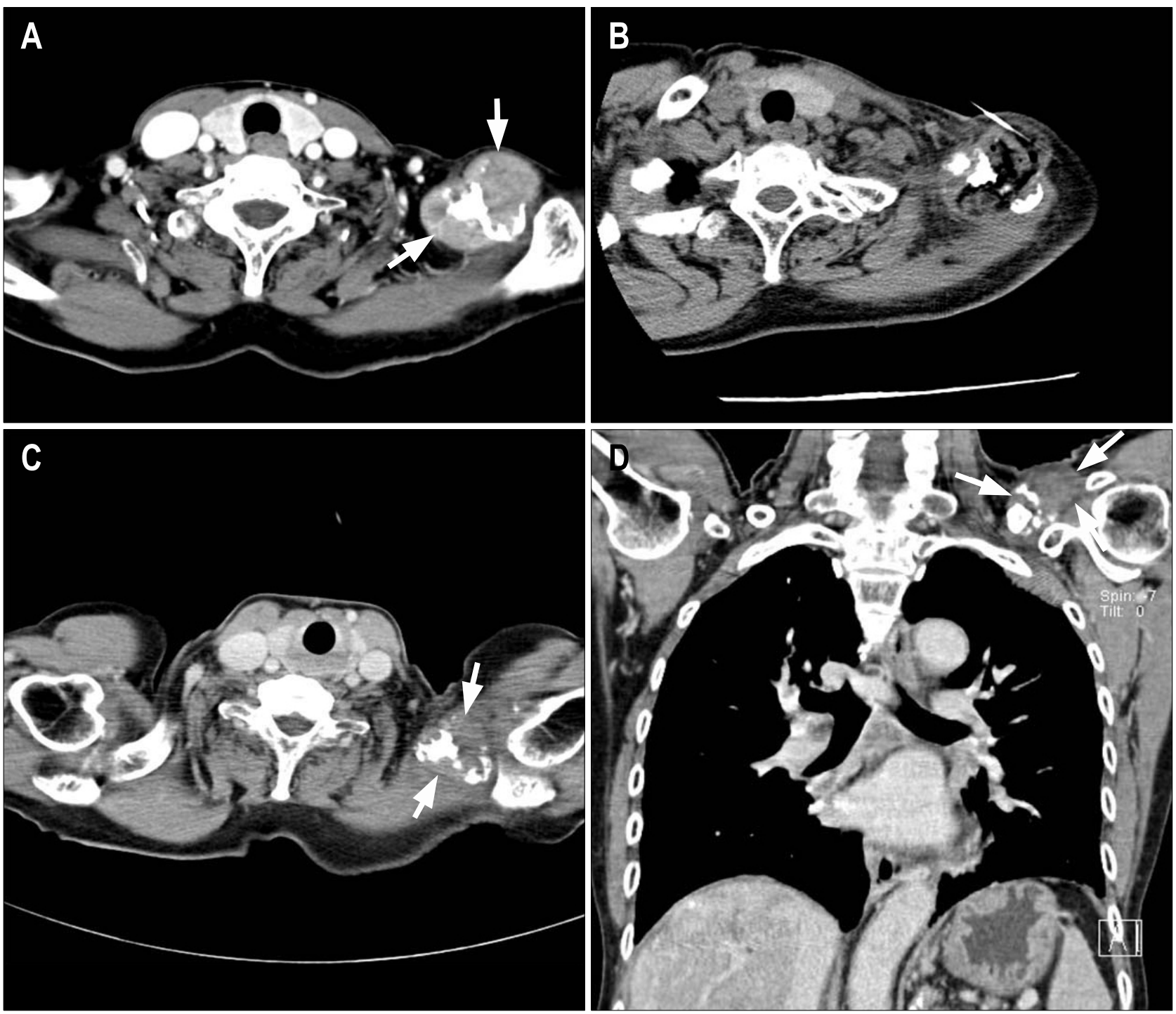

Fig. 3. Computed tomography (CT)-guided high-dose percutaneous ethanol injection (PEI) therapy in a 75-year-old woman with left clavicle metastasis of hepatocellular carcinoma. (A) Contrast-enhanced neck CT image shows a large mass in the left clavicle (arrows). (B) CT-guided PEI therapy was performed for this bone metastasis at four sessions, with an average of $20 \mathrm{~mL}$ of $99 \%$ ethanol being injected during each session. (C, D) Contrast-enhanced axial (C) and coronal (D) neck CT images obtained 2 years after PEI therapy show complete necrosis of left-clavicle metastasis (arrows). 
otherwise treated surgically or with TACE who present with adverse prognostic factors or risks for these therapies, and may be an option for selected patients with advanced disease previously excluded from any therapy. PEI therapy can be performed in an attempt to decrease total tumor burden for palliative purpose. In limited cases, complete necrosis may be possible. ${ }^{23,24}$ In several articles, the multipronged PEI therapy has it possible to treat medium and large HCCs under conscious sedation in less than three injection sessions. ${ }^{10,11}$

\section{HCC with distant metastasis}

If extrahepatic lesion discovers at imaging, either curative surgical therapy or percutaneous ablation therapy may not be offered and the patient will be left with only palliation. ${ }^{25-27}$ Natsuizaka et al. $^{28}$ reported that the prognosis was very poor in patients with HCC with extrahepatic metastases, and the median survival period and 1-year survival rate of those patients were 7 months and $24.9 \%$, respectively. Removal of extrahepatic metastasis of HCC may offer a chance for long-term survival in selected patients without poor prognostic factors (Child Pugh class $\mathrm{B}$ and $\mathrm{C}$, high alphafetoprotein, metastases to multiple organs) and whose intrahepatic tumors are controllable. ${ }^{28}$ Removal of a solitary extrahepatic metastasis may be justified provided that intrahepatic recurrence is well controlled by locoregional therapy. ${ }^{29}$ PEI therapy has been performed for the treatment of adrenal metastasis, ${ }^{12}$ nodal metastasis, ${ }^{13}$ and bone metastasis from HCC in some institutions. Our experience in bone metastases showed that, of 15 patients with 22 bone metastases undergone PEI therapy, complete necrosis was noted in 12 patients and partial necrosis was noted in 3 patients, and bone pain was completely relieved in all patients 2 days after the last session of PEI therapy, and overall survival rates at 1-year and 2-year were $62 \%$ and $33 \%$, respectively (Fig. 3). Our experience in adrenal metastases showed that, of 10 patients with adrenal metastases undergone PEI therapy, complete necrosis was noted in 8 patients and partial necrosis was noted in 2 patients, and overall survival rates at 1-year and 2-year were $58 \%$ and $33 \%$, respectively (unpublished data; presented at Society of Gastrointestinal Intervention, 2009).

In any injection therapy the fluid spreads in an unpredictable way but confines within the mass and ablates tumor tissue. In contrast, RFA generates heat around the radiofrequency electrode tip, which is distributed homogeneously in all directions, and ablates tissue around tip, irrespective of tumor tissue or normal structure. ${ }^{1}$ As most metastatic HCCs usually have adjacent vital structures, PEI therapy would be safer than RFA.

\section{HCC in patients with poor hepatic function}

Local ablation therapy is contraindicated in patients with gross ascites and coagulopathy that cannot be corrected. PEI therapy should not be performed with uncorrected prothrombin times in excess of 16 seconds, or with a platelet count less than 40,000 per cubic $\mathrm{mm}^{9}$ However, because PEI is less traumatic and carries no risk of damage to the remaining parenchyma, PEI therapy is safer than any other treatment modality of HCC. 3 Even in Child-Pugh $\mathrm{C}$ class patients, PEI therapy may be performed.

\section{CONCLUSION}

High-dose PEI therapy is safe and effective for the treatment of HCCs at risk or difficult to ablate with RFA. This procedure contributes to the combination therapy with TACE or RFA in advanced staged HCC, and also to the treatment of large HCC or extrahepatic metastasis from HCC. This role of PEI therapy should be stressed for the treatment of HCCs in proper clinical situations.

\section{REFERENCES}

1. Mahnken AH, Bruners P, Gunther RW. Local ablative therapies in HCC: percutaneous ethanol injection and radiofrequency ablation. Dig Dis 2009;27:148-156.

2. Shiina S, Teratani T, Obi S, Hamamura K, Koike Y, Omata M. Nonsurgical treatment of hepatocellular carcinoma: from percutaneous ethanol injection therapy and percutaneous microwave coagulation therapy to radiofrequency ablation. Oncology 2002;62(Suppl 1):64-68.

3. Livraghi T. Radiofrequency ablation, PEIT, and TACE for hepatocellular carcinoma. J Hepatobiliary Pancreat Surg 2003;10:67-76.

4. Garrean S, Hering J, Helton WS, Espat NJ. A primer on transarterial, chemical, and thermal ablative therapies for hepatic tumors. Am J Surg 2007;194:79-88.

5. Jansen MC, van Hillegersberg R, Chamuleau RA, van Delden OM, Gouma DJ, van Gulik TM. Outcome of regional and local ablative therapies for hepatocellular carcinoma: a collective review. Eur J Surg Oncol 2005;31: 331-347.

6. Lencioni R, Cioni D, Crocetti L, Bartolozzi C. Percutaneous ablation of hepatocellular carcinoma: state-of-the-art. Liver Transpl 2004;10(2 Suppl 1):S91-S97.

7. Livraghi T, Goldberg SN, Lazzaroni S, Meloni F, Solbiati L, Gazelle GS. Small hepatocellular carcinoma: treatment with radio-frequency ablation versus ethanol injection. Radiology 1999;210:655-661.

8. Poon RT, Fan ST, Tsang FH, Wong J. Locoregional therapies for hepatocellular carcinoma: a critical review from the surgeon's perspective. Ann Surg 2002;235:466-486.

9. Livraghi T. Role of percutaneous ethanol injection in the treatment of hepatocellular carcinoma. Dig Dis 2001;19: 
292-300.

10. Kuang M, Lu MD, Xie XY, et al. Ethanol ablation of hepatocellular carcinoma Up to $5.0 \mathrm{~cm}$ by using a multipronged injection needle with high-dose strategy. Radiology 2009;253:552-561.

11. Ho CS, Kachura JR, Gallinger S, et al. Percutaneous ethanol injection of unresectable medium-to-large-sized hepatomas using a multipronged needle: efficacy and safety. Cardiovasc Intervent Radiol 2007;30:241-247.

12. Shibata T, Maetani Y, Ametani F, Itoh K, Konishi J. Percutaneous ethanol injection for treatment of adrenal metastasis from hepatocellular carcinoma. AJR Am J Roentgenol 2000;174:333-335.

13. Zuo CJ, Wang PJ, Shao CW, et al. CT-guided percutaneous ethanol injection with disposable curved needle for treatment of malignant liver neoplasms and their metastases in retroperitoneal lymph nodes. World J Gastroenterol 2004; 10:58-61.

14. Redvanly RD, Chezmar JL, Strauss RM, Galloway JR, Boyer TD, Bernardino ME. Malignant hepatic tumors: safety of high-dose percutaneous ethanol ablation therapy. Radiology 1993;188:283-285.

15. Takayasu K, Muramatsu Y, Asai S, Kobayashi T. CT fluoroscopy-assisted needle puncture and ethanol injection for hepatocellular carcinoma: a preliminary study. AJR Am J Roentgenol 1999;173:1219-1224.

16. Joseph FB, Baumgarten DA, Bernardino ME. Hepatocellular carcinoma: CT appearance after percutaneous ethanol ablation therapy. Work in progress. Radiology 1993;186: 553-556.

17. Shibata T, Iimuro Y, Yamamoto Y, et al. CT-guided transthoracic percutaneous ethanol injection for hepatocellular carcinoma not detectable with US. Radiology 2002;223: 115-120.

18. Sato M, Watanabe Y, Tokui K, Kawachi K, Sugata S, Ikezoe J. CT-guided treatment of ultrasonically invisible hepatocellular carcinoma. Am J Gastroenterol 2000;95: 2102-2106.

19. Rhim H, Goldberg SN, Dodd GD 3rd, et al. Essential techniques for successful radio-frequency thermal ablation of malignant hepatic tumors. Radiographics 2001;21 Spec No:S17-S35.

20. Bartolozzi C, Lencioni R, Caramella D, et al. Treatment of large HCC: transcatheter arterial chemoembolization combined with percutaneous ethanol injection versus repeated transcatheter arterial chemoembolization. Radiology 1995; 197:812-818.

21. Kirikoshi H, Saito S, Yoneda M, et al. Outcome of transarterial chemoembolization monotherapy, and in combination with percutaneous ethanol injection, or radiofrequency ablation therapy for hepatocellular carcinoma. Hepatol Res 2009;39:553-562.

22. Zhang YJ, Liang HH, Chen MS, et al. Hepatocellular carcinoma treated with radiofrequency ablation with or without ethanol injection: a prospective randomized trial. Radiology 2007;244:599-607.

23. Livraghi T, Benedini V, Lazzaroni S, Meloni F, Torzilli G, Vettori C. Long term results of single session percutaneous ethanol injection in patients with large hepatocellular carcinoma. Cancer 1998;83:48-57.

24. Dettmer A, Kirchhoff TD, Gebel M, et al. Combination of repeated single-session percutaneous ethanol injection and transarterial chemoembolisation compared to repeated single-session percutaneous ethanol injection in patients with non-resectable hepatocellular carcinoma. World J Gastroenterol 2006;12:3707-3715.

25. Katyal S, Oliver JH 3rd, Peterson MS, Ferris JV, Carr BS, Baron RL. Extrahepatic metastases of hepatocellular carcinoma. Radiology 2000;216:698-703.

26. Tanaka O, Kanematsu M, Kondo H, et al. Solitary mediastinal lymph node metastasis of hepatocellular carcinoma: MR imaging findings. Magn Reson Imaging 2005;23:111114.

27. Uenishi $\mathrm{T}$, Hirohashi $\mathrm{K}$, Shuto $\mathrm{T}$, et al. The clinical significance of lymph node metastases in patients undergoing surgery for hepatocellular carcinoma. Surg Today 2000;30: 892-895.

28. Natsuizaka M, Omura T, Akaike T, et al. Clinical features of hepatocellular carcinoma with extrahepatic metastases. J Gastroenterol Hepatol 2005;20:1781-1787.

29. Poon RT, Fan ST, O'Suilleabhain CB, Wong J. Aggressive management of patients with extrahepatic and intrahepatic recurrences of hepatocellular carcinoma by combined resection and locoregional therapy. J Am Coll Surg 2002; 195:311-318. 